\title{
Ergebnisse zur Gesamtüberlebenszeit aus der Phase III BREAK-3-Studie mit Dabrafenib
}

\author{
GSK gab aktualisierte Resultate für Tafinlar (Dabrafenib) aus einer geplanten Analyse der Phase III \\ BREAK-3-Studie bei 250 Patienten mit BRAF V600E mutiertem metastasiertem Melanom bekannt. Die \\ Ergebnisse, die neue Überlebensdaten inkludieren, zeigen, dass $45 \%$ der Patienten, die mit Dabrafenib \\ behandelt wurden, nach zwei Jahren noch immer am Leben waren [1]. Die Daten wurden auf dem Kongress

\section{der European Society for Medical Oncology (ESMO) Ende September 2014 in Madrid präsentiert.}

Quelle: GSK - GlaxoSmithKline Pharma GmbH

Literatur:

1. Hauschild A, Grob JJ, Demidov LV et al (2014) ABSTRACT 1092: „An Update on Overall Survival (OS) and Follow-On Therapies in BREAK-3, a Phase III, Randomized Trial: Dabrafenib (D) vs. Dacarbazine (DTIC) in Patient (pts) with BRAF V600E Mutation-Positive Metastatic Melanoma (MM)." European Society for Medical Oncology (ESMO) Congress

2. Hauschild A, Grob JJ, Demidov LV et al (2012) Dabrafenib in BRAF-mutated metastatic melanoma: a multicentre, openlabel, phase 3 randomised controlled trial. Lancet 380:358-365

3. Tafinlar EU Summary of Product Characteristics, 30 August 2013. Accessed here: http://www.ema. europa.eu/docs/en_GB/ document_library/EPAR_ Summary_for_the_public/human/002604/ WC500149674.pdf
Über die Analyse des primären Endpunktes, progressionsfreies Überleben (progression free survival - PFS), wurde bereits 2012 berichtet [2]. Die jetzt veröffentlichten Ergebnisse beziehen sich auf einen sekundären Endpunkt der Studie; die abschließende Analyse der Überlebensdaten (overall survival - OS) wird für 2016 erwartet.

$45 \%$ der Patienten, die nur mit Dabrafenib behandelt wurden, waren nach zwei Jahren noch am Leben - verglichen mit $32 \%$ der Patienten, die eine Behandlung mit Dacarbazin (DTIC) begonnen hatten. $59 \%$ der Patienten mit DTIC-Behandlung, deren Erkrankung sich verschlechterte, erhielten in der Folge Dabrafenib und sind in den Resultaten des DTIC-Kontrollarms inkludiert. Während dieser Therapiewechsel Patienten im Kontrollarm den potentiellen Vorteil eines experimentellen Wirkstoffes sichert, kann sich ein Einfluss auf die vergleichenden Studienresultate ergeben, da Patienten, die beide Präparate erhielten, im DTIC Studienarm inkludiert werden.
Beim geplanten Zweijahres-FollowUp zeigte die Studie eine mediane Überlebensrate (OS) von 20,0 Monaten für den Dabrafenib-Arm (95\% Cl 16,8-24,4) verglichen mit 15,6 Monaten für den DTIC-Arm (95\% Cl 12,7-21,2) [Risikoquotient (HR) 0,77 (95\% Cl 0,52-1,13) - nicht statistisch signifikant.] [1]

„Wir sind durch die $45 \%$ Überlebensrate mit Dabrafenib nach zwei Jahren bestärkt", sagte Dr. Paolo Paoletti, Präsident Onkologie, GSK. „Die Behandlung von Melanomen hat sich in den letzten Jahren stark weiterentwickelt und wir sehen jetzt die Vorteile, die zielgerichtete Therapie den richtigen Patienten bringen kann."

Das in dieser Analyse beobachtete Sicherheitsprofil von Dabrafenib [3] war konsistent mit dem Sicherheitsprofil, das bei der ersten Analyse von BREAK-3 beobachtet wurde. Die fünf häufigsten Nebenwirkungen (adverse events - AE) bei mit Dabrafenib behandelten Patienten waren Hyperkeratose (41\%), Arthralgie (37\%), Kopfschmerzen (36\%), Fieber $(33 \%)$ und Alopezie (29\%).

\section{Die BREAK-3-Studie}

BREAK-3 (BRF113683) ist eine Phase III, randomisierte, open-label-Studie, die Wirksamkeit, Sicherheit und Verträglichkeit von Dabrafenib und DTIC bei Patienten mit fortgeschrittenem (Stadium III) oder metastasiertem (Stadium IV) Melanom und BRAF V600E Mutation vergleicht. Patienten mit unbehandeltem, BRAF V600E mutationspositivem, metastasiertem Melanom erhielten nach Zufallsprinzip entweder Dabrafenib (150 mg zwei Mal am Tag, oral) oder DTIC $(1000 \mathrm{mg} / \mathrm{m}$ intravenös alle drei Wochen).

Der primäre Endpunkt der Studie war progressionsfreies Überleben, sekundäre vorab spezifizierte Endpunkte inkludierten OS (overall survial). Die primäre Analyse der Studie wurde bereits 2012 publiziert [2].

\section{hautnah $2014 \cdot 13: 4$}

DOI 10.1007/s12326-014-0134-0

Online publiziert: 22. Oktober 2014 C) Springer-Verlag Wien 2014 